\title{
Time-Optimal Attitude Control Scheme for a Spinning Missile
}

\author{
E. Jahangir* and R. M. Howe $\dagger$ \\ University of Michigan, Ann Arbor, Michigan 48109
}

\begin{abstract}
The problem of minimum-time attitude control of a spinning missile is addressed. The missile is modeled as a rigid body that is symmetric about its spin axis. A single reaction jet provides the necessary transverse moments. The missile is assumed to have some arbitrary initial transverse angular velocity, and it is desired to take it to some final attitude in minimum time while reducing the transverse angular velocity to zero. A scheme to generate thruster firing times as functions of the initial and desired states of a spinning missile is described. This scheme involves transforming the state variables and integrating the transformed state and costate equations backward in time and generating the control history using the properties of the optimal control. The control history, given in terms of the thruster firing times, is stored as a function of the boundary conditions. A feedback control law based on function generation is then proposed that uses only the first thruster turn-on and turn-off times. This proposed control scheme can be implemented in real time with good accuracy.
\end{abstract}

\section{Introduction}

$\mathbf{O}$ VER the past three decades many papers and reports have treated various aspects of homing schemes and trajectory control associated with these schemes. Most of these papers consider surface-to-air or air-to-air missiles that use aerodynamic forces for trajectory control. With the advent of the strategic defense initiative, much attention has been focused on the interception of satellites or intercontinental ballistic missiles outside the sensible atmosphere. Hence, aerodynamic forces cannot be generated for vehicle control. Instead, the thrust of a rocket engine is used to provide the necessary maneuver forces, with vehicle attitude control employed to point the thrust in the desired direction. Conventional thrust vector control systems tend to add both weight and complexity, and as a result counter the objective of minimizing the weight of the guided warhead. The simplest control involves a single thruster at right angles to the spin axis of the missile. In this scheme the missile is given a large roll rate and the thruster is turned on for a fraction of each revolution in the roll and at the right time during each roll cycle so that the desired attitude changes are achieved. Meanwhile the main thruster, by producing a thrust component perpendicular to the flight path, provides the necessary trajectory changes.

The problem of attitude control of spinning rigid bodies has not received much attention recently, although some research had been reported on this topic in the 1960s. Athans and Falb ${ }^{1}$ considered the problem of time-optimal velocity control of a rotating body with a single axis of symmetry. However, they did not mention the complete attitude reorientation problem. Howe $^{2}$ proposed an attitude control scheme for sounding rockets that uses a single control jet. The control jet is fired for a fixed duration whenever certain conditions on direction cosines or transverse angular velocity are satisfied. This results in the alternate reduction of attitude error and transverse angular velocity, finally ending in a limit cycle. References 3-11 discuss the problem of reorienting a rotating rigid body that has no initial transverse angular velocity. Windenknecht ${ }^{3}$ proposed a simple system for the sun orientation of spinning satellites. In this scheme the desired attitude is achieved by a

Received Aug. 9, 1990; revision received Jan. 30, 1992; accepted for publication Feb. 29, 1992. Copyright (C) 1992 by the American Institute of Aeronautics and Astronautics, Inc. All rights reserved.

*Consultant R\&D, 10 Papri Road, Wah Cannt. 47040, Pakistan. Member AIAA.

$\dagger$ Professor, Aerospace Engineering, 324 AEB. Associate Fellow AIAA. succession of 180 deg precessional motions, each resulting in a small attitude change (small-angle approximations are assumed valid), until the spin axis arrives at an attitude corresponding to the dead zone of the sun sensors. Cole et al. ${ }^{4}$ prescribed the desired attitude change and solved for the necessary torques, but gave no details on mechanization. Other papers that have proposed active attitude control systems for spin-stabilized vehicles have been published by Adams, ${ }^{5}$ Freed, ${ }^{6}$ and Grasshoff, ${ }^{7}$ but none of these has explicitly discussed the reorientation problem. Grubin ${ }^{8}$ used the concept of finite rotations to mechanize a two-impulse scheme for reorienting the spin axis of a vehicle. If the torques are ideally impulsive, then the scheme is theoretically perfect. However, in the case of finite-duration torquing, considerable errors can result. Wheeler ${ }^{10}$ extended Grubin's work to include asymmetric spinning satellites, but the underlying philosophy is the same. Porcelli and Connolly ${ }^{11}$ used a graphical approach to obtain control laws for the reorientation of a spinning body. By assuming small angles and small angular velocities, they linearized the system equations and proved that a two-impulse control scheme is fuel-optimal. Two suboptimal control laws were then derived for the case of limited thrust based on the two-impulse solution. Most recently, Jahangir and Howe ${ }^{12}$ have proposed a time-optimal scheme that does not require solving a two-point boundary-value problem (TPBVP). This scheme can be used for the specific case when only two thruster firings are sufficient to complete the time-optimal attitude change maneuver. If the boundary conditions happen to lie outside this subset of the state space, the algorithm given in Ref. 12 fails to converge, since a two-pulse time-optimal solution does not exist for such a case. If a control law is desired for boundary conditions that require more than two thruster pulses, we must solve a TPBVP involving 10 nonlinear differential equations.

Both the two-pulse and the multiple-pulse solutions require iterations and, therefore, can be costly in terms of the computer time required for the solution to converge and also in terms of the complexity of the iterative update scheme. Hence, an online iterative procedure does not appear to be practical for a real-time control algorithm. One possible alternative is to precompute the thruster firing times by solving a TPBVP for discrete values of the desired boundary conditions. These thruster firing times can then be stored as a table in an onboard computer and the control scheme implemented in real time by a table look-up and interpolation.

Since we must store the control history as a function of the boundary conditions, we look for ways to generate a set of boundary condition points for which the thruster firing times are known without solving an iterative problem. To this end a 


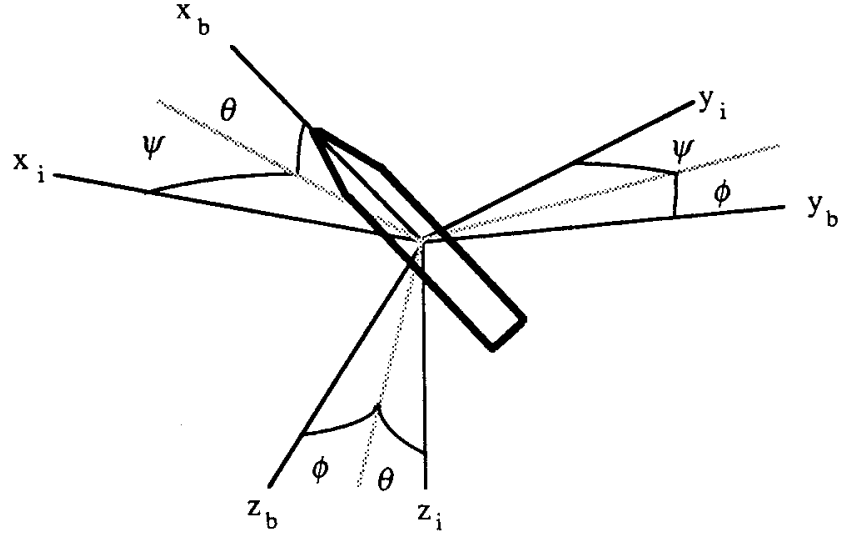

Fig. 1 Axis systems.

new state vector is introduced in Sec. III that is related to the original state vector by transformation. We will show in Secs. IV and V that we can generate a trajectory on the boundary of the set of reachable states by assuming a set of final conditions and integrating the transformed state and costate equations backward in time. Since the boundary of the set of reachable states defines all of the minimum-time trajectories, we can obtain all of the desired boundary conditions and associated time-optimal control histories by varying the final conditions over the range of their possible values. Finally, in Sec. VI we present a control scheme based on function generation. In this scheme table look-up followed by interpolation is used to determine the required thruster firing times. It is shown that this proposed control scheme can be implemented in real time with good accuracy.

\section{Equations of Motion}

Figure 1 shows the orientation of the moving body axes $x_{b}$, $y_{b}, z_{b}$ relative to the inertial reference axes $x_{i}, y_{i}, z_{i}$, and also the Euler angles $\psi, \theta, \phi$ relating the two axis systems. The body axes origin is at the missile's center of gravity (c.g.), with the $x_{b}$ axis assumed to be the axis of symmetry; the $y_{b}$ and $z_{b}$ axes lie in a plane perpendicular to the longitudinal axis $x_{b}$. The missile is modeled as a rigid cylindrical body. We also assume that the control jet is located in the $x_{b}-z_{b}$ plane and pointed in the direction of the $z_{b}$ axis. When fired, the control jet generates a constant positive moment about the $y_{b}$ axis.

We have assumed no disturbances such as aerodynamic forces, gravity, solar radiation pressures, or structural damping. Because of the short flight times, these disturbances have a negligible effect on the dynamics of the missile. Since no moment is applied about the $x_{b}$ axis and $I_{y}=I_{z}$ (the moments of inertia about the $y_{b}$ and $z_{b}$ axes are equal for a missile that is axially symmetric about its $x_{b}$ axis), it turns out that $\omega_{x}$, the missile angular velocity component along the $x_{b}$ axis, is a constant equal to the initial spin velocity of the missile. We then obtain a set of five state equations: two dynamical equations involving the transverse angular velocities and three kinematical equations giving the rates of change of Euler angles.

We let $\omega_{x}, \omega_{y}$, and $\omega_{z}$ denote the angular velocities about the $x_{b}, y_{b}$, and $z_{b}$ axes, respectively. Also, $I_{x}, I_{y}$, and $I_{z}$ denote the moments of inertia of the missile about its $x_{b}, y_{b}$, and $z_{b}$ axes, respectively. The moment applied about the $y_{b}$ axis is represented by $M_{y}$. We define the following dimensionless variables:

$$
\begin{array}{cc}
\Omega_{y}=\frac{\omega_{y}}{\omega_{x}}, \quad \Omega_{z}=\frac{\omega_{z}}{\omega_{x}}, & \lambda_{y}=\frac{M_{y}}{I_{y} \omega_{x}^{2}} \\
A=1-\frac{I_{x}}{I_{y}}=1-\frac{I_{x}}{I_{z}}, & T=\omega_{x} t
\end{array}
$$

We also define the - operator as differentiation with respect to the dimensionless time $T$. The equations of motion can now be written as

$$
\begin{gathered}
\dot{\Omega}_{y}=A \Omega_{z}+\lambda_{y} \\
\dot{\Omega}_{z}=-A \Omega_{y} \\
\dot{\psi}=\left(\Omega_{y} \sin \phi+\Omega_{z} \cos \phi\right) \sec \theta \\
\dot{\theta}=\Omega_{y} \cos \phi-\Omega_{z} \sin \phi \\
\dot{\phi}=1+\left(\Omega_{y} \sin \phi+\Omega_{z} \cos \phi\right) \tan \theta
\end{gathered}
$$

where $\psi, \theta$, and $\phi$ represent the Euler angles corresponding to yaw, pitch, and roll, respectively.

To write a state variable description of the system, we define the state $\boldsymbol{x}$ of the system as

$$
\boldsymbol{x}=\left[\begin{array}{lllll}
\Omega_{y} & \Omega_{z} & \psi & \theta & \phi
\end{array}\right]^{T}
$$

and the control $u$ as

$$
u=\lambda_{y}
$$

Equations (1-5) can now be written in standard form

$$
\dot{x}=f(x)+g u
$$

where

$$
f(x)=\left[\begin{array}{c}
A x_{2} \\
-A x_{1} \\
\left(x_{1} \sin x_{5}+x_{2} \cos x_{5}\right) \sec x_{4} \\
x_{1} \cos x_{5}-x_{2} \sin x_{5} \\
1+\left(x_{1} \sin x_{5}+x_{2} \cos x_{5}\right) \tan x_{4}
\end{array}\right]
$$

We assume that at the initial time, the missile-body axis system coincides with the inertial axis system. The initial transverse angular velocity of the missile, however, is nonzero. We thus obtain the following initial condition:

$$
\boldsymbol{x}_{0}=\left[\begin{array}{lllll}
x_{1,0} & x_{2,0} & 0 & 0 & 0
\end{array}\right]^{T}
$$

We want to find a control that will take this initial state to a desired state, described by some nonzero desired yaw and pitch angles and zero final transverse angular velocity, in minimum time. The desired final state vector $\boldsymbol{x}_{d}$ can be written as

$$
x_{d}=\left[\begin{array}{lllll}
0 & 0 & x_{3, d} & x_{4, d} & \text { free }
\end{array}\right]^{T}
$$

We also assume an upper bound $u_{\max }$ on the control $u$. Thus, the constraint on the control can be written as

$$
0 \leq u \leq u_{\max }
$$

The numerical values for the two parameters $A$ and $u_{\max }$ which will be used later in examples, are

$$
A=0.9, \quad u_{\max }=0.02
$$

This value of $A$ corresponds to a length-to-diameter ratio of 3.775 for a cylindrical body of uniform density. A missile weighing $10 \mathrm{lb}$ and having a uniform mass density of aluminum would have the following dimensions:

$$
\text { length }=12.30 \text { in. }, \quad \text { diameter }=3.26 \text { in } .
$$

If the moment arm is half the length and the spin velocity 50 $\mathrm{rad} / \mathrm{s}, u_{\max }=0.02$ corresponds to a thrust of $2.79 \mathrm{lb}$. 


\section{Time-Optimal Control Formulation}

It can be shown that the solution of the time-optimal problem, as given in Sec. II, involves a TPBVP. The solution of a TPBVP requires iterations and, therefore, is difficult to implement in real time. For this reason, the missile thruster firing times are computed offline and stored as a table in an onboard computer. Function generation is then used to compute the thruster turn-on and turn-off times online as functions of the boundary conditions.

Instead of obtaining the thruster switch times by solving this iterative problem, we consider a different approach. An alternative optimal control formulation in terms of a new state vector is given. It is shown that, by assuming a set of final conditions and integrating backward in time, we can generate time-optimal trajectories in the state space.

We define a new reference axis system. This axis system is fixed in the target and its $x$ axis points along the desired direction of the missile $x_{b}$ axis. The orientation of the missile with respect to an observer fixed in the target is given by the Euler angles $y_{3}, y_{4}$, and $y_{5}$, which correspond to yaw, pitch, and roll, respectively. We also define $y_{1}=x_{1}$ and $y_{2}=x_{2}$. Thus, we can write a new state vector

$$
\boldsymbol{y}=\left[\begin{array}{lllll}
y_{1} & y_{2} & y_{3} & y_{4} & y_{5}
\end{array}\right]^{T}
$$

The two state vectors $x$ and $y$ are related to a transformation (see Sec. V.C for the transformation formulas). The equations of motion can be written in terms of this new state vector and are given by

$$
\dot{y}=f(y)+g u
$$

We assume the initial and final conditions, respectively, to be

$$
\begin{gathered}
y\left(T_{0}\right)=y_{0}=\left[\begin{array}{lllll}
y_{1,0} & y_{2,0} & y_{3,0} & y_{4,0} & y_{5,0}
\end{array}\right]^{T} \\
y\left(T_{f}\right)=y_{f}=\left[\begin{array}{lllll}
0 & 0 & 0 & 0 & \mathrm{free}^{T}
\end{array}\right]^{T}
\end{gathered}
$$

A time-optimal control problem can be formulated for this system. We want to minimize the maneuver time so that we can write the performance index as

$$
J=\int_{T_{0}}^{T_{f}} 1 \mathrm{~d} t
$$

under the constraints of Eqs. (11) and (12).

This is one specific case of a general Mayer problem. Filippov ${ }^{13}$ gives a theorem and proves the existence of an optimal control for a Mayer problem. At this time, no general theorems are available on the uniqueness of optimal solutions for the one-sided controls, i.e., $0 \leq u \leq u_{\max }$. Therefore, we can only give necessary conditions for $u^{*}$ to be an optimal control.

Proceeding with the derivation of the necessary conditions on the time-optimal control, we write the Hamiltonian

$$
H=q^{T} \dot{y}-1
$$

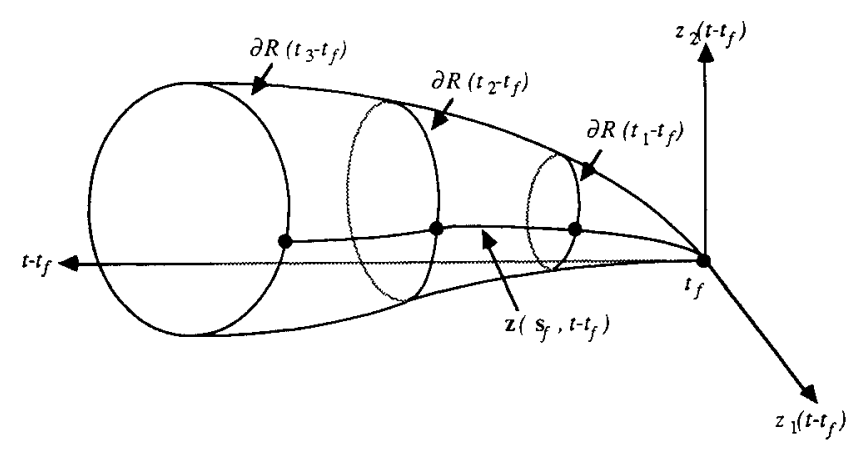

Fig. 2 Set of reachable states for a two-dimensional system. where $q$ is the costate vector. The necessary conditions for $u^{*}$ to be an optimal control are

$$
\begin{gathered}
\dot{y}^{*}=\frac{\partial H}{\partial q}=f\left(y^{*}\right)+g u^{*} \\
\dot{q}=-\frac{\partial H}{\partial y}=H\left(y^{*}\right) q^{*} \\
u^{*}= \begin{cases}u_{\max } & \text { if } q_{1}^{*}>0 \\
0 & \text { if } q_{1}^{*}<0\end{cases}
\end{gathered}
$$

where $H(y)=-\partial f / \partial y$. Equations (17) and (18) are the differential equations for the state and costate vector and Eq. (19) is derived from the optimality condition, i.e., maximizing the Hamiltonian $H$ with respect to the control $u$.

The boundary conditions on the state variables are given by Eqs. (13) and (14). The boundary conditions on the Hamiltonian and the costate variables are derived from the transversality conditions

$$
\begin{gathered}
H\left(T_{f}\right)=0 \\
q\left(T_{0}\right)=q_{0}=[\text { free free free free free }]^{T} \\
q\left(T_{f}\right)=q_{f}=[\text { free free free free } 0]^{T}
\end{gathered}
$$

We note from the theory of necessary conditions that

$$
\frac{\partial H\left(y^{*}, q^{*}, T\right)}{\partial T}=\frac{d H\left(y^{*}, q^{*}, T\right)}{\mathrm{d} T}=0
$$

This, in addition to Eq. (20), shows that

$$
H\left(y^{*}, q^{*}, T\right)=0 \quad \text { for all } \quad T \in\left[T_{0}, T_{f}\right]
$$

We observe that this formulation still results in a TPBVP. If the initial state vector $y\left(T_{0}\right)$ is specified, an initial costate vector $q\left(T_{0}\right)$ must be determined that results in the desired final state and costate vectors. However, the state and costate vectors at the final time have some simple features. Each of the components of these vectors is either zero or free. Therefore, it is worthwhile to examine the system characteristics if the state and costate equations are integrated backward in time starting at $T_{f}$.

In the next section we discuss a two-dimensional system in terms of some simple sets in the state and costate space. By looking at the problem from a geometric point of view, we show for this two-dimensional system that the origin can be connected to all points in the set of reachable states in minimum time by varying the costate vector over $R^{2}$ and integrating the system equations backward in time.

\section{Set of Reachable States for a Two-Dimensional System}

In this section we examine the characteristics of the following two-dimensional system:

$$
\dot{z}=h(z, u)
$$

where $z$ is a $2 \times 1$ state vector and $u$ the scalar control. The desired final condition is assumed to be the origin, i.e., $z\left(t_{f}\right)=0$. If we subject the system with final state $z\left(t_{f}\right)=0$ to all control histories and integrate the system backward in time starting at $t_{f}$, we obtain a set of states that are reachable from the origin at time $t-t_{f}$, or simply the set of reachable states. We denote the set of reachable states as $R\left(t-t_{f}\right)$ in Fig. 2. In the figure the boundary of the set of reachable states at time $t_{i}-t_{f}$ is denoted by $\partial R\left(t_{i}-t_{f}\right)$. It is well known from the geometric properties of the optimal control that the boundary of the set of reachable states defines all of the minimum-time solutions. We, therefore, conduct the following experiment. 
Let $u_{\min }(z, s)$ be the optimal control that is obtained by minimizing the Hamiltonian with respect to the control, where $s$ is the $2 \times 1$ costate vector. Hence, if $z$ is an optimal motion, it satisfies

$$
\dot{z}^{*}=h\left[z^{*}, u_{\min }\left(z^{*}, s^{*}\right)\right]
$$

where $s^{*}$ is a solution to the related costate equations. Clearly, $z^{*}\left(t_{f}\right)=0$.

To obtain a specific time-optimal trajectory, we need to assume some final conditions on the costate vector. We let $s\left(t_{f}\right)=s_{f}$, where $s_{f}$ is an arbitrary constant vector. The system equations $\dot{z}=h\left[\left(z, u_{\text {min }}(z, s)\right]\right.$ can now be integrated backward in time starting from the final time $t_{f}$ to obtain a trajectory $z\left(s_{f}, t-t_{f}\right)$ shown in Fig. 2 . The trajectory $z\left(s_{f}, t-t_{f}\right)$ represents the fact that it is a function of the specified final costate vector $s_{f}$ and time parameter $t-t_{f}$. This trajectory connects all points along its path to the origin in minimum time. Also, as mentioned earlier, this minimum-time trajectory lies on the boundary of the set of reachable states. By storing $z\left(s_{f}, t-t_{f}\right)$ at discrete points in time, we can obtain a set of points in the state space for which the time-optimal control history is known. If we assume a different initial $\boldsymbol{s}_{f}$, another time-optimal trajectory is obtained. By varying $s_{f}$ over $R^{2}$ and integrating the system for each $s_{f}$ backward in time, all trajectories on the boundary of the set of reachable states can be generated. In this way, all of the reachable states can be obtained at discrete intervals and the associated control history stored as a function of these states.

In the next section we apply the technique just described to the complete nonlinear minimum-time attitude control problem of a spinning missile. By using the procedure analogous to this section, all minimum-time trajectories can be generated. By storing the state vector at discrete points in time along the minimum-time trajectories, we are able to generate a set of points for which the thruster firing times are known.

\section{Examination of the $y$ System}

We use here the idea of backward integration and examine the system behavior. In an example similar to that in Sec. IV, we conduct the following experiment.

Four of the final state variables are zero, as given in Eq (14). We assume that $y_{5}\left(T_{f}\right)=y_{5, f}$, where $y_{5, f}$ is an arbitrary constant. Similarly, we assume an arbitrary value for the final costate vector $q\left(T_{f}\right)=q_{f}$, where $q_{5, f}=0$. The expression for the time-optimal control is given in Eq. (19). Once all of the final conditions on the state and costate variables are specified, we start the integration at $T_{f}$ and integrate the state and costate equations backward in time. At each numerical integration step, we obtain a $y\left(T^{\prime}\right)$, where $T^{\prime}=T-T_{f}$. This trajectory connects all points along its path to the specified final state in minimum time. By varying the first four components of the final costate vector $\boldsymbol{q}_{f}$ over $\boldsymbol{R}^{4}$ [the fifth component is zero as given in Eq. (22)], the entire boundary of the set of reachable states is generated, starting from the specified $y_{f}$. We note here that the only nonzero component of the state vector is $y_{5, f}$, which corresponds to the roll angle of the missile. This angle can take on values in the range $[-\pi, \pi]$. By varying $y_{5, f}$ in this range and following the aforementioned procedure of generating the boundary of the set of reachable states, all the time-optimal solutions can be generated. During the integration, the state vector $y$ and corresponding thruster switch times can be stored at discrete points in time. Hence, this procedure gives a set of $\boldsymbol{y}$ points for which the control history, given in terms of the thruster firing times, is known.

The control scheme can then be implemented in real time by using the thruster switch times which are stored at discrete values of the transformed state vector $y$. However, in the actual missile the desired attitude is measured with respect to the moving missile frame, whereas the vector $y$ gives the orientation of the missile with respect to an observer fixed in the target. Hence, it is desirable to store the boundary condi- tions in terms of the original state vector $\boldsymbol{x}$. The boundary conditions in terms of the $\boldsymbol{x}$ vector are given in Eqs. (9) and (10). The state vector $y\left(T^{\prime}\right)$ can be transformed back to our original system to obtain the corresponding boundary conditions $x_{1,0}, x_{2,0}, x_{3, d}$, and $x_{4, d}$.

The procedure to generate the control history as a function of the boundary conditions can be summarized in the following way:

1) Initialize $\boldsymbol{y}_{f}$ and $q_{f}$.

2) Integrate the $\dot{y}$ and $\dot{q}$ equations backward in time and at each $T_{n}^{\prime}=n \Delta T$ obtain $y\left(T_{n}^{\prime}\right)$, where $\Delta T$ is the time interval chosen to give desired data point spacing between $y\left(T_{n}^{\prime}\right)$ and $y\left(T_{n+1}^{\prime}\right)$, and $n$ is a positive integer. Note that the numerical integration step can be a submultiple of $\Delta T$.

3) Transform $y\left(T_{n}^{\prime}\right)$ to obtain the boundary conditions in the original form $x_{1,0}, x_{2,0}, x_{3, d}$, and $x_{4, d}$. Store the thruster switching times as functions of these four variables. Note that if $q_{1}>0$, then $T_{1}=0$, and similarly if $q_{1}<0$ then $T_{1}>0$, where $T_{1}$ is the first turn-on time of the thruster.

Each of these steps is discussed in the following sections.

\section{A. Initialization of $y_{f}$ and $q_{f}$}

As indicated earlier, five of the variables at $T_{f}$ are zero

$$
y_{1, f}=y_{2, f}=y_{3, f}=y_{4, f}=q_{5, f}=0
$$

The other five variables at $T_{f}$ are free. These must be varied over all possible values to obtain the optimal control history as a function of the boundary conditions. The variable $y_{5, f}$ corresponds to the roll angle and, thus, is confined to

$$
y_{5, f} \in[-\pi, \pi]
$$

The space over which $q_{1, f}, q_{2, f}, q_{3, f}$, and $q_{4, f}$ must be varied is a subset of the costate space. We refer to this subspace as $\mathcal{Q}$. Definition: The space $\mathcal{Q}$ is defined as

$$
\begin{aligned}
& \mathcal{Q}=\left\{q\left(T_{f}\right): q_{5}\left(T_{f}\right)=0, q_{i}\left(T_{f}\right)= \pm 1, q_{j}\left(T_{f}\right) \in[-1,+1],\right. \\
& \quad i, j=1,2,3,4, j \neq i\}
\end{aligned}
$$

An algorithm to vary these variables over the range of their possible values is given here

$$
\begin{array}{ll}
\text { do } y_{5, f}=-\pi, \pi, \delta & \begin{array}{l}
\text { where } \delta \text { is the desired } \\
\text { spacing between values } \\
\text { of } y_{5, f}
\end{array} \\
\text { do } \quad \begin{array}{l}
i=1,4 \\
q_{i, f}= \pm 1 \\
\text { do } q_{j, f}=-1,+1, \Delta
\end{array} & \begin{array}{l}
\text { where } j=1,2,3,4, \\
j \neq i, \text { and } \Delta \text { is the de- } \\
\text { sired spacing between } \\
\text { values of } q_{j, f}
\end{array}
\end{array}
$$

During the implementation of our scheme to generate timeoptimal solutions, we observe that a constant $\Delta$ (uniform spacing in $q_{j, f}$ ) does not result in a uniform span of the entire desired space of time-optimal solutions. We find that when $q_{j, f}$ is close to zero, very small $\Delta$ is needed to span the set of desired time-optimal solutions. Conversely, when $q_{j, f}$ is not close to zero, $\Delta$ need not be small.

\section{B. Integration of State and Costate Equations}

A practical issue in the implementation of the scheme given in this section is the choice of a numerical integration algorithm and the handling of discontinuities that occur when the control switches from on to off or vice versa.

The RK-4 fixed-step algorithm is used to integrate the state and costate equations. We utilize the fact that analytic solutions for $y_{1}$ and $y_{2}$ can be obtained from Eq. (12). Thus, the equations for $\dot{y}_{1}$ and $\dot{y}_{2}$ do not have to be integrated numerically. The analytical solutions for $y_{1}$ and $y_{2}$ are also used to 
Table 1 Transformation relations between $x$ and $y$

\begin{tabular}{cc}
\hline $\boldsymbol{x}$ & $\varphi(y)$ \\
\hline$x_{1,0}$ & $y_{1}$ \\
$x_{2,0}$ & $y_{2}$ \\
$x_{3, d}$ & $\tan ^{-1}\left(\frac{-\sin y_{3} \cos y_{5}+\cos y_{3} \sin y_{4} \sin y_{5}}{\cos y_{3} \cos y_{4}}\right)$ \\
$x_{4, d}$ & $-\sin ^{-1}\left(\sin y_{3} \sin y_{5}+\cos y_{3} \sin y_{4} \cos y_{5}\right)$ \\
\hline \hline
\end{tabular}

obtain the half- and full-frame derivative estimates of the remaining state and costate variables, as required in the RK-4 integration algorithm.

There is a discontinuity in these derivatives when the application of the control $u$ starts or stops. This switching time is a function of $q_{1}$, the first component of the costate vector, as given by Eq. (19). If this discontinuity occurs within an integration step, it can cause large errors in the numerical solution. To reduce these errors, the step size must be chosen small enough to meet some integration error criterion, which can result in excessive computational time. Several papers have been written suggesting special methods to circumvent this difficulty. We choose the method proposed by Howe et al. ${ }^{14}$ for its accuracy and ease of implementation. In this scheme, at each successive time step, $q_{1}$ is tested to see whether it has switched sign. If it has not, the integration proceeds to the next step. If switching has occurred, the time of its occurrence is computed by combining a fixed-point simplified Hermite interpolation with a continued fraction formula. Hermite interpolation is also used to compute the state and costate variable values at the crossover time. The RK-4 algorithm is then used to integrate through the remainder of the fixed-time step.

\section{Transformation Relations Between $x$ and $y$}

Let us consider a vector $r$ originating at the missile c.g. We let $\left\{r_{m}\right\}$ represent the components of the vector $r$ in the missile-body axis frame; $\left\{r_{t}\right\}$ the components of the same vector in the frame 3 ; and $\left\{r_{f}\right\}$ the components of the same vector in the frame $\mathcal{F}$. The frame of reference $J$ is an arbitrary axis system whose $x$ axis coincides with the desired direction of the missile $x_{b}$ axis. The frame of reference $\mathfrak{F}$ is defined to be the missile axis system at the end of the maneuver. Previously, we have defined $x_{3, d}$ and $x_{4, d}$ to be the yaw and pitch angles describing the orientation of the desired direction of the missile $x_{b}$ axis with respect to the missile-body axis system. In addition, we define $x_{5, d}$ to be the roll angle of the frame $\mathcal{F}$ with respect to the missile-body axis system. It should be borne in mind that $x_{5, d}$ is free in the description of the optimal control problem in Sec. II. Thus, we can write

$$
\left\{r_{f}\right\}=\left[C\left(x_{3, d}, x_{4, d}, x_{5, d}\right)\right]\left\{r_{m}\right\}
$$

where $[C]$ is the direction cosine matrix and is defined in the following way:

$$
[C(\psi, \theta, \phi)]=\left[\begin{array}{c}
\cos \psi \cos \theta \\
-\sin \psi \cos \phi+\cos \psi \sin \theta \sin \phi \\
\sin \psi \sin \phi+\cos \psi \sin \theta \cos \phi
\end{array}\right.
$$

We have already defined $y_{3}=y_{3}\left(T-T_{f}\right), y_{4}=y_{4}\left(T-T_{f}\right)$, and $y_{5}=y_{5}\left(T-T_{f}\right)$ to be the Euler angles describing the missile orientation relative to an observer fixed in the frame $\mathfrak{J}$. Therefore, we can write

$$
\left\{r_{m}\right\}=\left[C\left(y_{3}, y_{4}, y_{5}\right)\right]\left\{r_{t}\right\}
$$

Finally, the frame $\mathfrak{F}$ is obtained by rotating the frame $\mathfrak{J}$ about its $x$ axis. This rotation is given by the angle $y_{5, f}=y_{5}\left(T_{f}\right)$ and we get

$$
\left\{r_{f}\right\}=[\Phi]\left\{r_{t}\right\}
$$

where

$$
[\boldsymbol{\Phi}]=\left[\begin{array}{ccc}
1 & 0 & 0 \\
0 & \cos y_{5, f} & \sin y_{5, f} \\
0 & -\sin y_{5, f} & \cos y_{5, f}
\end{array}\right]
$$

The matrices $[C]$ and $[\Phi]$ are both direction cosine matrices; therefore, each is an orthogonal matrix. Hence, the inverse of these matrices is obtained by merely transposing them.

Equations (25) and (26) can be combined to write

$$
\left\{r_{f}\right\}=[\Phi]\left[C\left(y_{3}, y_{4}, y_{5}\right)\right]^{T}\left\{r_{m}\right\}
$$

Comparing Eqs. (28) and (23), we finally get

$$
\left[\boldsymbol{C}\left(x_{3, d}, x_{4, d}, x_{5, d}\right)\right]=[\Phi]\left[C\left(y_{3}, y_{4}, y_{5}\right)\right]^{T}
$$

By equating the entries of the matrices on the left- and righthand sides of Eq. (29), we can obtain expressions for $x_{1,0}, x_{2,0}$, $x_{3, d}$, and $x_{4, d}$ in terms of $y\left(T-T_{f}\right)$ and $y_{5}\left(T_{f}\right)$ without any ambiguity in the quadrants.

Table 1 summarizes the transformation relations between the boundary conditions, given in terms of the state vector $\boldsymbol{x}$, and the new state vector $y$ at time $T-T_{f}$.

\section{Mechanization of the Control Scheme}

The thruster switch times $T_{i}, i=1,2, \ldots, n$, can be obtained from the approach given in Sec. $V$ for the desired set of boundary conditions, where $T_{i}$ is the time when control switches and $n$ is the total number of switches required to complete the attitude change maneuver. To implement this scheme in real-time, the switch times $T_{i}, i=1,2, \ldots, n$, must be stored in an onboard computer. We propose a control scheme that only needs to store $T_{1}$ and $T_{2}$, the first turn-on and turn-off times, respectively.

In this scheme table look-up followed by interpolation is used to compute $T_{1}$ and $T_{2}$. The thruster is then turned on from $T_{1}$ to $T_{2}$. After this first thruster firing has been completed, we can measure the state variables at $T_{2}$. The switch times $T_{1}$ and $T_{2}$ can now be recomputed based on this measured state. These new $T_{1}$ and $T_{2}$ correspond to the old $T_{3}$ and $T_{4}$, respectively, for the previous $T_{1}$ and $T_{2}$. Thus for the two-pulse case, the new $T_{2}=T_{f}$, the time at which the target is reached in the ideal case. In the presence of interpolation, modeling, and measurement errors, there will still be a finite error in the state at $T_{2}$. For reasonable modeling and measurement errors, it is felt that this final state error will be small enough to fall within the target tolerance for switchover to a

$\left.\begin{array}{cc}\sin \psi \cos \theta & -\sin \theta \\ \cos \psi \cos \phi+\sin \psi \sin \theta \sin \phi & \cos \theta \sin \phi \\ -\cos \psi \sin \phi+\sin \psi \sin \theta \cos \phi & \cos \theta \cos \phi\end{array}\right]$

linear control law, perhaps using pulse-width modulation. If the state error at $T_{2}$ is outside the linear control-law region, table look-up based on the state at $T_{2}$ will compute $T_{3}$ and $T_{4}$ (actually a new $T_{1}$ and $T_{2}$ ) with $T_{4}-T_{3}$ small, such that the target state is more closely realized after the $T_{3}, T_{4}$ pulse. The process will continue to converge until the error in terminal 
state becomes small enough to switch over to the linear control law. When more than two pulses are required initially in the ideal case, the time-optimal control will eventually reach the two-pulse solution region in state space, after which the foregoing convergence argument applies.

When we store the thruster times at discrete points in time while integrating the state and costate equations backward in time, a function with randomly spaced input values is obtained. However, it is desirable for real-time function generation to have equally spaced function input variables. The function table of equally spaced input values can be obtained by interpolation across the randomly spaced input values. The most widely used interpolation method in the flight simulation industry is piecewise multilinear interpolation. ${ }^{15}$ However, this method cannot be used when the input values are randomly spaced. Instead, piecewise linear interpolation ${ }^{16}$ must be employed to obtain a function with equally spaced inputs from a function with unequally spaced inputs. Once the function with equally spaced inputs is created, table search and multilinear interpolation can be carried out in real time, as described by Gilbert. ${ }^{15}$

Function values are stored at discrete values of the input variables $x_{1,0}, x_{2,0}, x_{3, d}$, and $x_{4, d}$. When using these "rectangular" coordinates as the input parameters, the grid size remains constant, independent of the distance from the origin at $x_{1,0}=x_{2,0}=x_{3, d}=x_{4, d}=0$. However, as we move away from the origin, the accuracy requirements decrease. Thus, we need not use a closely spaced grid when far away from the origin. To satisfy this requirement, we introduce "spherical" coordinates in the following way:

$$
\begin{gathered}
R=\sqrt{4 \pi^{2}\left(x_{1,0}^{2}+x_{2,0}^{2}\right)+x_{3, d}^{2}+x_{4, d}^{2}} \\
\beta=\tan ^{-1}\left(\frac{x_{1,0}}{x_{2,0}}\right) \\
\gamma=\tan ^{-1}\left(\frac{\left.2 \pi \sqrt{x_{1,0}^{2}+x_{2,0}^{2}}\right)}{x_{3, d}}\right) \\
\zeta=\tan ^{-1}\left[\frac{\sqrt{4 \pi^{2}\left(x_{1,0}^{2}+x_{2,0}^{2}\right)+x_{3, d}^{2}}}{x_{4, d}}\right]
\end{gathered}
$$

These relations are derived from a simple extension of the three-dimensional spherical coordinates. The transverse angular velocities are multiplied by $2 \pi$ so that they are the same order of magnitude as the angles.

The spherical coordinates $\beta, \gamma$, and $\zeta$ have the desired variable spacing. As we get farther away from the origin (increasing $R$ ), the spacing between the breakpoint values in $\beta$, $\gamma$, and $\zeta$ increases. It is desirable to have the same kind of

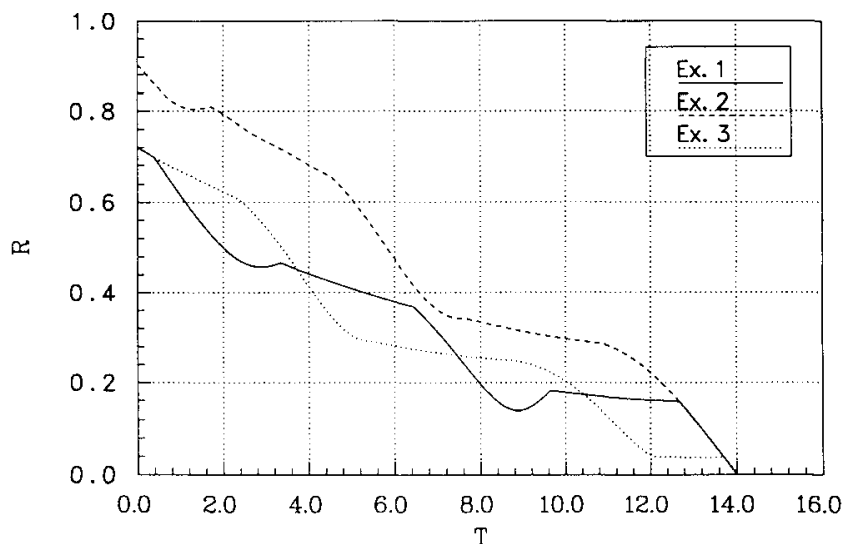

Fig. 3 Total error $R$ vs $T$.

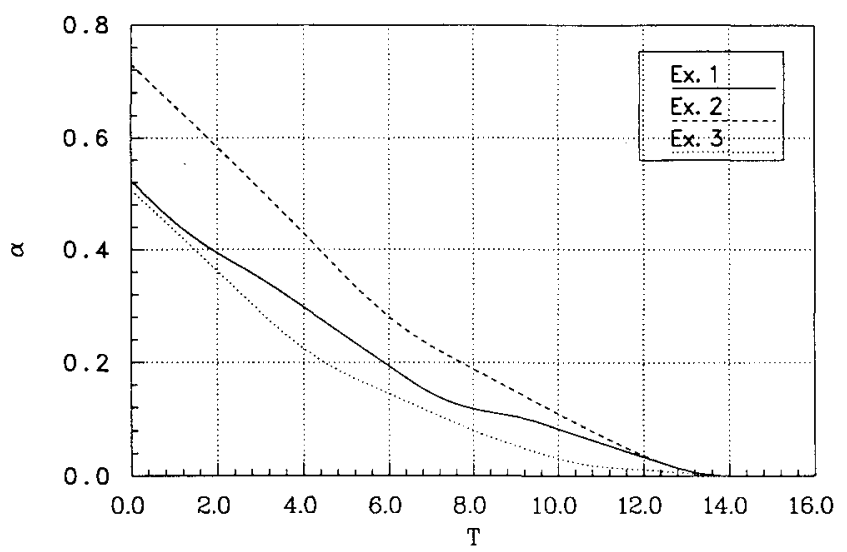

Fig. 4 Total angle $\alpha$ vs $T$.

spacing between discrete values of $R$. To this end, we store function values at equally spaced discrete values of $\sqrt{R}$ instead of $R$. Thus, close to the origin the function values are stored at small intervals to satisfy the higher accuracy requirements. When $R$ becomes relatively large, the accuracy requirements decrease and this is reflected by increased spacing between the stored function values.

Instead of storing $T_{1}$ and $T_{2}$, the first turn-on and turn-off times, we store $T_{1}$ and $\Delta T_{1}=T_{2}-T_{1}$. The variable $T_{1}$ is the first turn-on time as before and $\Delta T_{1}$ the duration of the thruster firing. This choice results in a smoother behavior of $T_{1}$ and $\Delta T_{1}$ as functions of the independent variables.

We can now write $T_{1}$ and $\Delta T_{1}$ as functions of the four input variables as follows

$$
\begin{aligned}
T_{1} & =f_{1}(w, x, y, z) \\
\Delta T_{1} & =f_{2}(w, x, y, z)
\end{aligned}
$$

where

$$
\begin{array}{rr}
w=\sqrt{R}, & x=\beta \\
y=\gamma, & z=\zeta
\end{array}
$$

The functions $f_{1}$ and $f_{2}$ are stored at the breakpoint values $w_{i}$, $x_{j}, y_{k}$, and $z_{l}$, which are, respectively, $I, J, K$, and $L$ in number and $\Delta w, \Delta x, \Delta y$, and $\Delta z$ apart.

In our working example, we find that

$$
\begin{aligned}
& \Delta w=2^{-5}=0.03125 \\
& \Delta x=\Delta y=\Delta z=\frac{\pi}{15}
\end{aligned}
$$

give satisfactory results. In addition, we choose $w_{0}=0.03125$.

We are only interested in a subset of the state space within which the time-optimal control is to be used. We define this subset by the requirement that during the maneuver $R$ cannot be greater than 1 rad. In practical situations, if the total error in $R$ is greater than $1 \mathrm{rad}$, it is improbable that the guidance scheme of the missile can home in on the target. The integer $I$ can now be computed and is the following:

$$
I=N I N T\left(\frac{w_{\max }}{\Delta w}\right)=\frac{\sqrt{1}}{0.03125}=32
$$

The integers $J, K$, and $L$ can be obtained from the fact that $x$, $y, z \in(0,2 \pi)$. Thus,

$$
J=K=L=\frac{2 \pi}{\pi / 15}=30
$$

The size of the array is determined from the integers $I, J, K$, and $L$ as follows:

$$
\text { size of array }=I \times J \times K \times L=864,000
$$


Table 2 Boundary conditions for the examples shown in Figs. 3-7

\begin{tabular}{lrrr}
\hline & \multicolumn{1}{c}{ Example 1 } & \multicolumn{1}{c}{ Example 2 } & Example 3 \\
\hline$R$ & $0.720006 E+00$ & $0.903172 E+00$ & $0.720303 E+00$ \\
$\beta$ & $0.371007 E+01$ & $0.543167 E+01$ & $0.139682 E+01$ \\
$\gamma$ & $0.231071 E+01$ & $0.882395 E+01$ & $0.100043 E+01$ \\
$\zeta$ & $0.197411 E+01$ & $0.232395 E+01$ & $0.989696 E+01$ \\
$x_{1,0}$ & $-0.419044 E-01$ & $-0.609231 E-01$ & $0.794368 E-01$ \\
$x_{2,0}$ & $-0.655966 E-01$ & $0.533560 E-01$ & $0.139611 E-01$ \\
$x_{3, d}$ & $-0.446497 E+00$ & $0.418599 E+00$ & $0.325083 E+00$ \\
$x_{4, d}$ & $-0.282582 E+00$ & $-0.617718 E+00$ & $0.395406 E+00$ \\
\hline
\end{tabular}

Table 3 Attitude errors at the end of three pulses when function generation is used to compute the thruster firing times

\begin{tabular}{lccc}
\hline \hline & Example 1 & Example 2 & Example 3 \\
\hline$R\left(T_{f}\right)$ & $0.367250 E-02$ & $0.189528 E-02$ & $0.397519 E-08$ \\
$\alpha\left(T_{f}\right)$ & $0.311947 E-02$ & $0.441320 E-03$ & $0.000000 E+00$ \\
$\Omega\left(T_{f}\right)$ & $0.308455 E-03$ & $0.293352 E-03$ & $0.994821 E-10$ \\
$T_{f}$ & $0.139966 E+02$ & $0.140026 E+02$ & $0.140100 E+02$ \\
\hline \hline
\end{tabular}

The precision associated with 16-bit fixed-point words should be more than adequate to represent the data in the function tables for $T_{1}$ and $\Delta T_{1}$. For the foregoing array size of 864,000 , this translates into a total storage requirement of less than 4 megabytes, which can easily be accomplished using eight currently available $16 \times 256 \mathrm{kbit}$ static ROM microchips. With any of a number of current microchip processors, the time required to calculate each pair of four-variable functions will be well under $100 \mu \mathrm{s}$. Thus, the control law as proposed can be mechanized at a $10 \mathrm{kHz}$ sample rate using computer hardware of negligible weight and cost.

\section{Example Trajectories}

We consider three examples here. The initial conditions and desired final conditions for the three examples are given in Table 2. In the exact solution the desired attitude is obtained at $T_{f}=14$ and requires three control pulses. We simulate the system using the proposed control scheme and compare the conditions at the end of three thruster firings with the exact solution.

Figure 3 shows the total error $R$, as defined in Eq. (30), as a function of dimensionless time $T$. We see that the attitude change maneuver is completed in about the same time as in the exact solution. As the missile $x_{b}$ axis moves toward the target direction, the angles $x_{3, d}$ and $x_{4, d}$ change with time. We define $\alpha=\cos ^{-1}\left(\cos x_{3, d} \cos x_{4, d}\right)$ as the total angular distance of the target direction with respect to the missile $x_{b}$ axis. Figure 4 shows this angle $\alpha$ as a function of the dimensionless time $T$. In Fig. 5, the position of the target direction relative to the moving missile-body axis system, as given by the yaw angle $x_{3, d}$ and pitch angle $x_{4, d}$, is plotted as the maneuver proceeds. An observer fixed in the missile body will see the target move in this fashion. The attitude change maneuver is completed when $x_{3, d}=x_{4, d}=0$.

The total transverse angular velocity $\Omega=\sqrt{x_{1}^{2}+x_{2}^{2}}$ is plotted as a function of the dimensionless time $T$ in Fig. 6 , where we recall that $x_{1}=\Omega_{y}$ and $x_{2}=\Omega_{z}$. Figure 7 shows the trajectories of $x_{1}$ and $x_{2}$ in the $x_{1}-x_{2}$ plane. When the radius given by $\Omega$ is constant, the missile coasts. Conversely, when the radius $\Omega$ changes, it means that the thruster is on.

In the exact solution three thruster firings are needed to bring the missile to its desired attitude. Table 3 summarizes the final attitude errors at the end of the third thruster firing when multivariable function generation is used to compute the thruster turn-on and turn-off times after each firing has been completed. In all three examples the final attitude is very close to the desired one. Once the error in attitude has been forced close to zero, it may be desirable to switch to some other control scheme. Frequently, when bang-bang control is em-

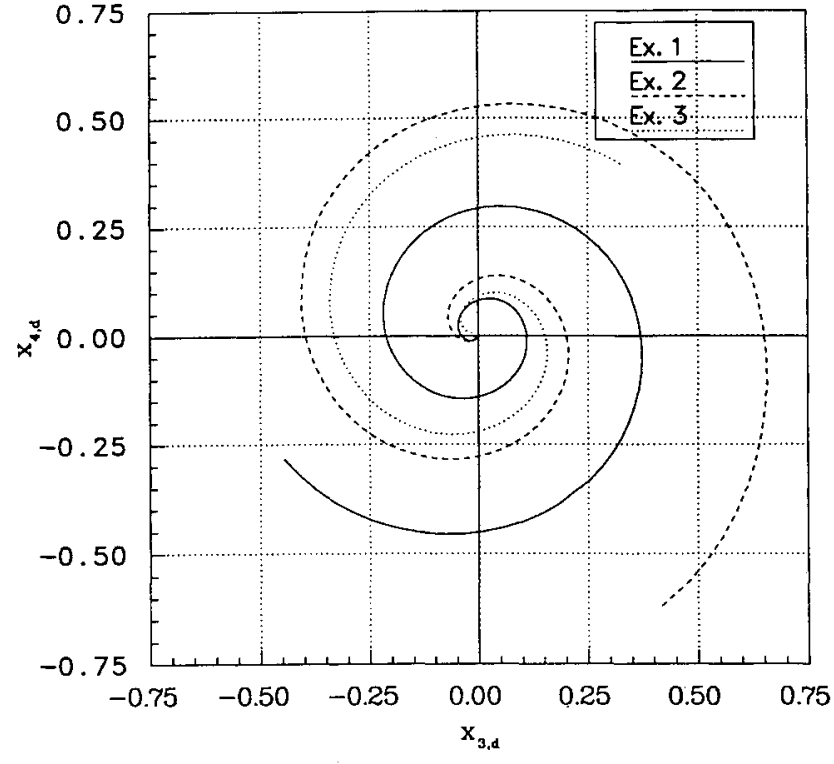

Fig. 5 Path of the target in the $x_{3, d}-x_{4, d}$ plane, where $x_{3, d}$ and $x_{4, d}$ are the yaw and pitch angles, respectively, of the desired pointing direction with respect to the moving missile-body axis frame.

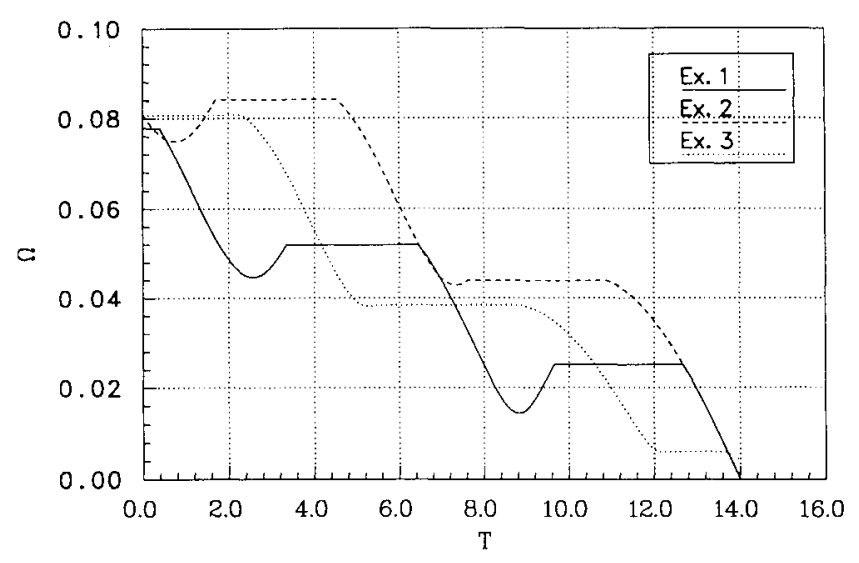

Fig. 6 Total transverse angular velocity $\Omega$ vs $T$.

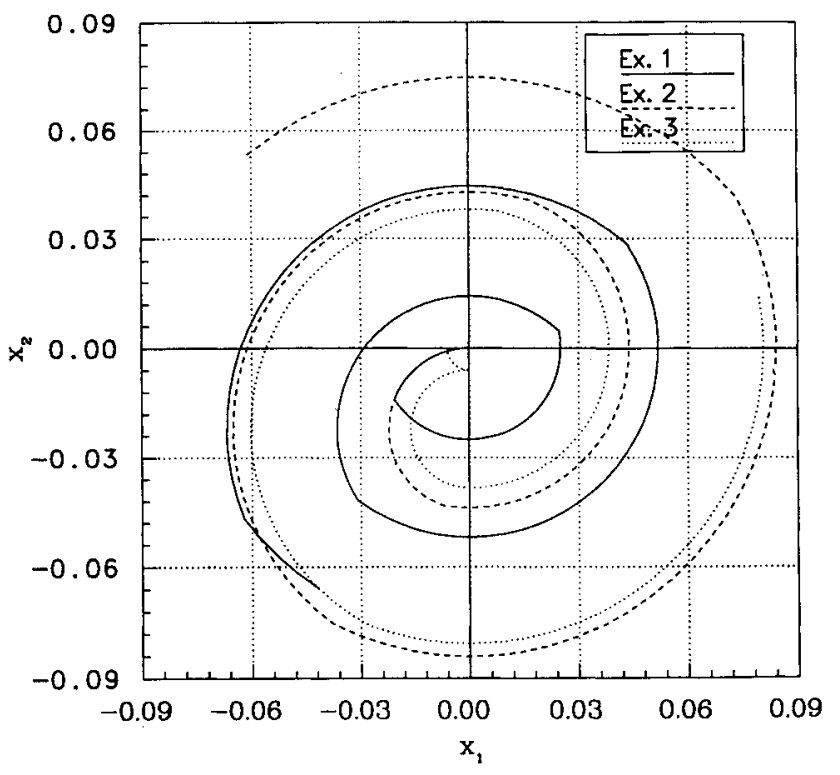

Fig. 7 Trajectories of transverse angular velocity components $x_{1}$ and $x_{2}$. 
ployed for error control, a linear control law is used close to the origin to avoid chattering. For the same reason, a linear control scheme can be used when the initial attitude of the missile is close to the desired one.

\section{Conclusion}

In this paper the problem of minimum-time attitude control of a spinning missile has been considered. Traditionally, this class of problems is handled by solving a two-point boundaryvalue problem. Instead, we have taken an alternative approach. This approach is based on offline computation of the thruster firing times, which are then stored in an onboard computer. Table look-up and interpolation are then used to determine the switch times online, with the thruster fired in accordance with the switch times thus obtained.

In the approach discussed in this paper, the state vector is transformed such that both the final desired attitude and attitude rate are zero. Then, for specific values of the final roll angle and costate vector, time-optimal solutions can be generated by numerically integrating the state and costate equations backward in time. Each set of final conditions yields a different time-optimal control. By varying the final conditions over the range of their possible values, all of the desired optimal control solutions can be generated. The boundary conditions and associated control history are stored at discrete points in time along the time-optimal trajectories. This set of points can be transformed to obtain a function table with equally spaced inputs. This procedure for generating a table of thruster turnon and turn-off times as functions of the boundary conditions results in a considerable reduction of the offline computational load.

Instead of storing all of the firing times, only the first thruster turn-on and turn-off times are saved. Based on the measured state after completion of this first pulse, new thruster turn-on and turn-off times are determined for the next pulse. Constant update of the state measurements used to determine the next thruster turn-on and turn-off times constitutes a feedback process that insures convergence to a near time-optimal solution, even in the presence of modeling and measurement errors. By employing standard search and interpolation routines, the required thruster firing times can be computed in real time. We also show that the final errors in attitude can be made very small if the spacing between the input-variable data points in the function table is chosen appropriately.

\section{Acknowledgments}

The research reported in this paper was supported by the U.S. Army Strategic Defense Command under Contract
DASG60-88-C-0037 and by the Air Force Office of Scientific Research under Contract F49620-86-C-0138.

\section{References}

${ }^{1}$ Athans, M., and Falb, P. L., Optimal Control, McGraw-Hill, New York, 1966, pp. 841-853.

${ }^{2}$ Howe, R. M., "Attitude Control of Rockets Using a Single Axis Control Jet," XIth International Astronautical Congress (Stockholm), 1960, pp. 88-98.

${ }^{3}$ Windenknecht, T. G., "A Simple System for Sun Orientation of a Spinning Satellite," Paper 61-204-1898, National IAS-ARS Joint Meeting, Los Angeles, June 1961.

${ }^{4}$ Cole, R. D., Ekstrand, M. E., and O'Neil, M. R., "Attitude Control of Rotating Satellites,'” ARS Journal, Vol. 31, No. 10, 1961, pp. 1446-1447.

${ }^{5}$ Adams, J. J., "Study of An Active Control System for a Spinning Body,"' NASA TN D-905, June 1961.

6Freed, L. E., "Attitude Control System for a Spinning Body," Paper 61-207-1901, National IAS-ARS Joint Meeting, Los Angeles, June 1961.

${ }^{7}$ Grasshoff, L. H., "A Method for Controlling the Attitude of a Spin-Stabilized Satellite,' ARS Journal, Vol. 31, No. 5, 1961, pp. 646-649.

${ }^{8}$ Grubin, C., "Generalized Two-Impulse Scheme for Reorienting a Spin Stabilized Vehicle," American Rocket Society Guidance, Control, and Navigation Conference, Stanford, CA, Aug. 1961.

${ }^{9}$ Patapoff, H., "Bank Angle Control System for a Spinning Satellite," AIAA Paper 63-339, Aug. 1963.

${ }^{10}$ Wheeler, P. C., "Two-Pulse Attitude Control of an Asymmetric Spinning Satellite," Guidance and Control II, Vol. 13, Progress in Astronautics and Aeronautics, AIAA, New York, 1964, pp. 261-287.

${ }^{11}$ Porcelli, G., and Connolly, A., "Optimal Attitude Control of a Spinning Space Body-A Graphical Approach," IEEE Transactions on Automatic Control, Vol. AC-12, No. 3, June 1967, pp. 241-249.

12Jahangir, E., and Howe, R. M., "A Two-Pulse Scheme for the Time-Optimal Attitude Control of a Spinning Missile,"' Proceedings of the AIAA Guidance, Navigation, and Control Conference (Portland, OR), AIAA, Washington, DC, Aug. 1990.

13Filippov, A. F., "On Certain Questions in the Theory of Optimal Control," SIAM Journal of Control, Vol. 1, No. 1, 1962, pp. 76-84.

${ }^{14}$ Howe, R. M., Ye, X. A., and Li, B. H., "An Improved Method for Simulation of Dynamic Systems with Discontinuous Nonlinearities," Transactions of the Society for Computer Simulation, Vol. 1, No. 1, Jan. 1984 , pp. 33-47.

${ }^{15}$ Gilbert, E. O., "A Special Digital Computer for High Speed Function Generation in Hybrid and Digital Simulation," Proceedings of the 8th AICA Congress, Delft, Aug. 1976.

${ }^{16}$ Weiser, A., and Zarantonello, S. E., "A Note on Piecewise Linear and Multilinear Table Interpolation in Many Dimensions," Mathematics of Computation, Vol. 50, No. 181, Jan. 1988, pp. $189-196$. 